\title{
Raman-Brillouin electronic density in short-period superlattices
}

\author{
Nicolas Large \\ Centre d'Elaboration de Matériaux et d'Etudes Structurales (CEMES), UPR 8011, CNRS-Université de Toulouse, 29 rue Jeanne Marvig, \\ BP 94347, 31055 Toulouse Cedex 4, France; \\ Centro Mixto de Física de Materiales, CSIC-UPV/EHU, Spain; \\ and Donostia International Physics Center (DIPC), Paseo Manuel de Lardizabal 5, 20018 Donostia-San Sebastián, Spain
}

Jean-Roch Huntzinger

Groupe d'Etude des Semiconducteurs (GES), UMR 5650, CNRS-Université Montpellier 2, Case Postale 074 Place Eugène Bataillon, 34095 Montpellier Cedex 5, France

Javier Aizpurua

Centro Mixto de Física de Materiales, CSIC-UPV/EHU, Spain and Donostia International Physics Center (DIPC), Paseo Manuel de Lardizabal 5, 20018 Donostia-San Sebastián, Spain

Bernard Jusserand*

Institut des NanoSciences de Paris (INSP), UMR 7588, CNRS-Université Pierre et Marie Curie, 140 Rue de Lourmel, 75015 Paris, France

\author{
Adnen Mlayah ${ }^{\dagger}$ \\ Centre d'Elaboration de Matériaux et d'Etudes Structurales (CEMES), UPR 8011, CNRS-Université de Toulouse, 29 rue Jeanne Marvig, \\ BP 94347, 31055 Toulouse Cedex 4, France
}

(Received 12 February 2010; revised manuscript received 7 May 2010; published 12 August 2010)

\begin{abstract}
We analyze and interpret resonant Raman-Brillouin scattering by folded acoustic vibrations in short-period GaAs/AlAs superlattices. Analysis of the spectra and their resonance behavior is performed using a RamanBrillouin electronic density constructed by combining thousands transitions between electronic eigenstates of the system according to their weight in the light-scattering process. We show that plots of this effective electronic density allow for capturing the essential physics of the electron-phonon interaction and of the resonant light-scattering process in a situation where complex effects are simultaneously present: electronic confinement in the quantum wells and wave-function delocalization due to interlayer coupling, folding of acoustic dispersion and symmetry changes in the deformation fields, resonant selection of optical transitions. Comparison between the measured spectra and those simulated using the Raman-Brillouin quantum model and the photoelastic model are presented. Activation and/or deactivation of the scattering by acoustic vibration doublets and changes in their intensity ratio with excitation energy are directly related to the Raman-Brillouin electronic density distribution along the superlattices axis. Limitations of the photoelastic model are discussed by comparing the steplike variation in the photoelastic coefficient to the Raman-Brillouin electronic density profiles.
\end{abstract}

DOI: $10.1103 /$ PhysRevB.82.075310

PACS number(s): 63.22.- m, 73.21.Cd, 73.22.Lp, 78.30.Fs

\section{INTRODUCTION}

Low-frequency Raman scattering in semiconductor superlattices (SLs) displays spectral features related to folding of acoustic phonons which originates in the periodic modulation of the acoustic and acousto-optic properties. This topic has been extensively investigated in the past ${ }^{1-5}$ and has recently regained a significant interest due to possible generation and detection of high-frequency coherent acoustic waves using femtosecond laser pulses. Applications in vibrational spectroscopy, nanoscale imaging of defects and picosecond modulation of semiconductors optoelectronic properties are targeted. In particular, a strong effort is devoted to the enhancement of the acousto-optic transduction efficiency in these devices using optical resonances. Most of the published works report Raman scattering by folded acoustic phonons recorded under nonresonant excitation conditions, ${ }^{1,2}$ i.e., with probe energies far from specific optical transitions of the system. These results were fairly well explained using the photoelastic model (PEM) which assumes a periodic steplike variation in the photoelastic coefficients along the SL axis: inside each layer the photoelastic coefficients are constant and their values in the bulk materials are used. ${ }^{3}$ This model does not take into account explicitly neither some size dependence of the photoelastic coefficients, nor some changes in the spatial dependence of the photoelastic function due to the electronic confinement. Therefore, it fails to describe the resonance behavior of the Raman scattering due to folded acoustic phonons. In order to overcome these limitations some energy dependence of the photoelastic coefficients can be introduced. However, this reproduces only homothetic variations in the Raman intensities of folded acoustic vibration modes.

Since the early work of Colvard et al. ${ }^{3}$ and Merlin et al. ${ }^{4}$ it has been shown that resonant Raman scattering by folded 
acoustic phonons in semiconductor SLs is properly interpreted using quantum mechanics: the inelastic lightscattering process is described within a third-order perturbation theory in which the resonantly excited optical transitions are taken into account explicitly. The acousto-optic interaction is mediated by the electronic states of the system owing to the electron-phonon and electron-photon interactions. ${ }^{6-8}$ Therefore, the resonant Raman spectra strongly reflect the electronic states selected by the optical excitation. In particular, the adequacy between the symmetry of the acoustic displacement field and that of the intermediate electronic states determines the Raman selection rules. However, when several electronic transitions are involved in the resonant lightscattering process, interpretation of the Raman features becomes difficult due to interferences between the different scattering paths. ${ }^{9}$ Moreover, the SL/substrate interface, the sample surface, ${ }^{10,11}$ thickness fluctuations of the SL layers ${ }^{12-14}$ and the presence of impurities may strongly affect the electronic states thus reflecting in the resonant Raman spectra. For these reasons, the relation between the electronic properties of a system and its resonant Raman spectra can be complex.

In previous works on quantum dots and quantum wells, some of us have introduced a Raman-Brillouin electronic density (RBED) by combining the electronic transitions and electronic states of a system according to their relative contribution to the light-scattering process. ${ }^{15,16}$ This approach allows to generate electronic density profiles that capture the physics of the electron-phonon interaction and related Raman-Brillouin spectra, even though thousands of electronic transitions may be involved. Indeed, the RBED is useful when a large number of electronic transitions are excited by the optical probe. This is for instance the case of semiconductor quantum dots excited close to $E_{1}$ transitions characterized by flat valence and conduction-band dispersions (i.e., large effective masses). ${ }^{15}$ In that situation, interferences between the numerous scattering paths take place, thus blurring the connection between the Raman-Brillouin spectral features and the excited electronic states. Moreover, it has been shown that the RBED is the link between the widely used photoelastic model of the Raman scattering and quantum models which accounts for optical resonance effects.

The present work is devoted to the introduction of the Raman-Brillouin electronic density for two-dimensional periodic nanostructures. The GaAs/AlAs short-period superlattices, investigated in this paper, are considered here as model systems on which the utility of the RBED can be tested. Direct comparison between simulated and measured spectra are presented. For each SL, the spatial distribution of the RBED is plotted for various excitation energies. It is shown how these RBED profiles, combined with the symmetry of the acoustic vibration modes, allow for a clear understanding of the resonant Raman-Brillouin scattering in a complex situation where periodicity, quantum confinement, layering of the vibrational properties and optical selection of electronic transitions are simultaneously present. Moreover, the RBED profiles are compared to the steplike profile of the Photoelastic coefficient. We show why the profile of this coefficient underestimates or overestimates the electron-phonon interaction thus leading to erroneous Raman-Brillouin scattering in-
TABLE I. Number of periods, nominal and measured thicknesses of the three superlattices.

\begin{tabular}{lccccc}
\hline \hline Sample & $\begin{array}{c}\text { Number } \\
\text { of } \\
\text { periods }\end{array}$ & $\begin{array}{c}d_{\mathrm{GaAs}} \\
\text { qanominal } \\
(\mathrm{nm})\end{array}$ & $\begin{array}{c}d_{\mathrm{GaAs}} \\
\text { measured } \\
(\mathrm{nm})\end{array}$ & $\begin{array}{c}d_{\mathrm{AlAs}} \\
\text { nominal } \\
(\mathrm{nm})\end{array}$ & $\begin{array}{c}d_{\mathrm{AlAs}} \\
\text { measured } \\
(\mathrm{nm})\end{array}$ \\
\hline SL1 & 200 & 2.954 & 2.90 & 1.173 & 1.25 \\
SL2 & 200 & 1.969 & 2.00 & 2.346 & 2.43 \\
SL3 & 200 & 0.985 & 0.99 & 3.519 & 3.55 \\
\hline \hline
\end{tabular}

tensities of folded acoustic phonons. The originality of the work lies in the introduction of the RBED as a theoretical tool to understand and interpret the main tendencies observed experimentally. We successfully describe variations in acoustic phonons doublets that could never be explained previously and are out of the scope of the standard photoelastic model.

\section{EXPERIMENTS AND STRUCTURES}

Three GaAs/AlAs superlattices with nearly the same nominal folding frequency $\left(d_{\mathrm{GaAs}} / v_{\mathrm{GaAs}}+d_{\mathrm{AlAs}} / v_{\mathrm{AlAs}}\right)^{-1}$ $=1.2 \mathrm{THz}$ and different GaAs $\left(d_{\mathrm{GaAs}}\right)$ and AlAs $\left(d_{\mathrm{AlAs}}\right)$ thicknesses are studied $\left(v_{\mathrm{GaAs}}\right.$ and $v_{\mathrm{AlAs}}$ are the longitudinal sound velocities in GaAs and AlAs). In Table I the nominal parameters as well as those deduced from the x-ray diffraction are shown. The measured thicknesses are very close to the nominal ones. It is well known that changing the well/ barrier thickness ratio in SLs leads to strong variations in the associated Raman signature (see Sec. III A). Our samples correspond to three different values of the well/barrier thickness ratio $\left(\frac{d_{\mathrm{GaAs}}}{d_{\mathrm{AIAs}}} \approx 2.32,0.82\right.$, and 0.28$)$ while the SLs period $d=d_{\mathrm{GaAs}}+d_{\mathrm{AlAs}}$ is almost the same (around $4.4 \mathrm{~nm}$ ). The samples were chosen to allow for a detailed investigation of their Raman-scattering properties covering the three situations $\frac{d_{\mathrm{GaAs}}}{d_{\mathrm{AlAs}}}<1, \frac{d_{\mathrm{GaAs}}}{d_{\mathrm{AlAs}}}=1$, and $\frac{d_{\mathrm{GaAs}}}{d_{\mathrm{AlAs}}}>1$.

Raman measurements have been performed at room temperature, in near-backscattering (BS) configuration using a Dilor XY800 triple spectrometer in the subtractive mode and a charge coupled device detector. Six excitation wavelengths from an argon ion laser and from a near infrared tunable Ti:Sa laser have been used. The Stokes Raman spectra, normalized either to the first (SL1) or to the second (SL2 and SL3) acoustic mode, are shown in Fig. 1. In agreement with the design of the samples (constant period), the average Raman shift of the acoustic phonon doublets is nearly the same for the three SLs and independent on the excitation energy; while the doublet splitting reflects the energy dependence of the phonon wave vector probed in BS and the slight variation in the average sound velocity from sample to sample. The relative intensity of the first and second doublets and of the two components of each doublet display strong variations from sample to sample: intensity ratio changes in the spectral components of the first acoustic doublet and scattering by the second acoustic doublet.

\section{MODELS}

The experimental results shown in Fig. 1 can be interpreted using either the PEM or the Raman-Brillouin quantum 


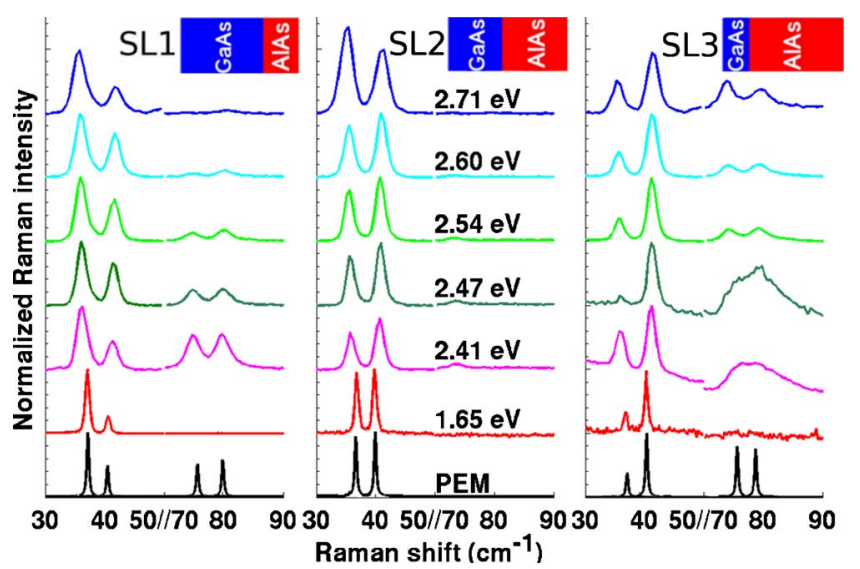

FIG. 1. (Color online) Color plots: Raman-Brillouin spectra recorded from the three superlattices for the excitation energies indicated in the central panel. The black spectra have been calculated using the PEM at $E_{i}=1.65 \mathrm{eV}$. The drawings at the top of the panels help to visualize the GaAs/AlAs thickness ratio.

model (RBQM). In the RBQM, the SL electronic eigenstates are taken into account explicitly.

\section{A. Photoelastic model}

Let us first briefly recall the photoelastic model. The PEM describes the light-scattering process in terms of modulation of the dielectric susceptibility by the vibration modes. Here, we discuss its integral form which has been widely used for the interpretation of the RB scattering in superlattices. ${ }^{2}$

For scattering by longitudinal-acoustic (LA) vibrations, the Raman-Brillouin intensity is given by ${ }^{2,17,18}$

$$
I_{\mathrm{RB}} \propto\left|\int A_{s}^{*}(z) A_{i}(z) P(z) \frac{\partial u_{m}(z)}{\partial z} d z\right|^{2},
$$

where $u_{m}$ is the displacement field along the $z$ direction for a LA mode $m, P(z)$ is the steplike periodic change in the Photoelastic coefficient along the superlattice axis given by $P(z)=0.48$ in GaAs and $P(z)=0.005$ in AlAs. ${ }^{3} A_{i}$ (respectively, $A_{s}$ ) is the potential vector of the incident (respectively, scattered) light. The displacement fields of LA vibrations are calculated as a linear combination of reflected and transmitted waves using the transfer-matrix method as described in Refs. 19 and 20. The displacement field $u_{m}(z)$ is normalized over the whole superlattice.

For an infinite superlattice, the dispersion of LA phonons along the superlattice axis is given by the well-known Rytov's formula 2,21

$$
\begin{aligned}
\cos (q d)= & \cos \left[\omega_{q}\left(\frac{d_{\mathrm{GaAs}}}{v_{\mathrm{GaAs}}}+\frac{d_{\mathrm{AlAs}}}{v_{\mathrm{AlAs}}}\right)\right] \\
& -\frac{\epsilon^{2}}{2} \sin \left(\omega_{q} \frac{d_{\mathrm{GaAs}}}{v_{\mathrm{GaAs}}}\right) \sin \left(\omega_{q} \frac{d_{\mathrm{AlAs}}}{v_{\mathrm{AlAs}}}\right),
\end{aligned}
$$

where $q$ is the LA phonons wavevector and $\epsilon$ $=\frac{\varrho_{\mathrm{GaAs}} v_{\mathrm{GaAs}}-\varrho_{\mathrm{AlAs}} v_{\mathrm{AlAs}}}{\left(\varrho_{\mathrm{GaAs}} v_{\mathrm{GaAs}} \varrho_{\mathrm{AlAs}} v_{\mathrm{AlAs}}\right)^{1 / 2}}$ is a parameter describing the contrast between the materials acoustic impedances. The LA phonons dispersion is shown in Fig. 2 for the three studied superlat-
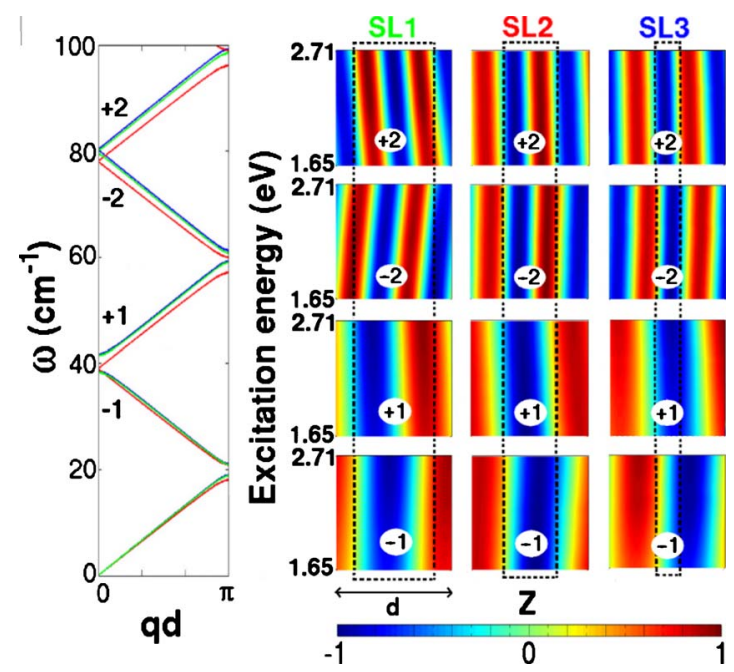

FIG. 2. (Color online) Left panel: dispersion of LA phonons for the superlattices, SL1 (green), SL2 (red), and SL3 (blue), calculated from Eq. (2). The acoustic branches are identified as $( \pm 1)$ and $( \pm 2)$. Right panels: deformation field $\operatorname{div}\left[u_{m}(z)\right]=\frac{\partial u_{m}(z)}{\partial z}$ of the modes $( \pm 1)$ and $( \pm 2)$, calculated using the transfer-matrix method for each finite superlattice and excitation energy. The deformations are shown within a unitary cell, centered on the GaAs layer. The black dashed lines indicate the GaAs/AlAs interfaces.

tices (considered as infinite).

For both PEM and RBQM, we assume incident (respectively, scattered) electromagnetic field $A_{i(s)}(z)$ of the form $\exp \left(i k_{i(s)} z\right)$, where $k_{i(s)}$ is the incident (respectively, scattered) photon wavevector component along the $z$ direction. The inplane component $\vec{k}_{\|}$of the incident and scattered wavevectors is here neglected.

In Fig. 1 the Raman-Brillouin spectra calculated using the PEM (Eq. (1)) are reported. The PEM spectra were generated with a transferred wave vector corresponding to the near infrared excitation $\left(E_{i}=1.65 \mathrm{eV}\right)$; the PEM intensities do not depend on the excitation energy. In contrast, it is very clear from the measured spectra (Fig. 1) that significant variations in the Raman intensities with excitation energy are observed and cannot be described by the photoelastic model. The most evident discrepancy is the absence of the second doublet in the near infrared spectra of SL1 and SL3 (red line in Fig. 1). Moreover, strong intensity variations in the second acoustic doublet and of the intensity ratio within the first acoustic doublet are observed depending on the excitation energy.

\section{B. Quantum model-Raman-Brillouin electronic density}

Here, we briefly recall the quantum theory of the resonant Raman-Brillouin scattering according to the approach reported in Refs. 2 and 22 and apply to this context the RBED introduced in Refs. 15 and 16.

In the frame of the quantum perturbation theory, the resonant Raman-Brillouin scattering is described as a three steps process in which the emission (or absorption) of a vibration mode occurs because of transitions between the electronic eigenstates of the system. In the case where the process is 
mediated by conduction electrons, the scattering efficiency is proportional to ${ }^{6-8}$

$$
\left|\sum_{e, e^{\prime}, h} \frac{\left\langle h\left|H_{e-p h t}^{s}\right| e^{\prime}\right\rangle\left\langle e^{\prime}\left|H_{e-v i b}\right| e\right\rangle\left\langle e\left|H_{e-p h t}^{i}\right| h\right\rangle}{\left(E_{s}-E_{e^{\prime}-h}+i \gamma_{e^{\prime}-h}\right)\left(E_{i}-E_{e-h}+i \gamma_{e-h}\right)}\right|^{2},
$$

where $e, e^{\prime}$ and $h$ are electron and hole eigenstates; $E_{e-h}$ and $\gamma_{e-h}$ are the energy and homogeneous broadening of the $e-h$ transition, respectively; $E_{e-h}$ is defined as: $E_{e-h}=E_{g}+E_{e}+E_{h}$ where $E_{e}$ (respectively, $E_{h}$ ) is the electron (respectively, hole) confinement energy and $E_{g}$ is the GaAs band-gap energy. $E_{i}$ and $E_{s}=E_{i} \pm \hbar \omega_{m}$ are, respectively, the incident and scattered photon energies $\left(\hbar \omega_{m}\right.$ being the energy of the absorbed or emitted vibration mode). The electronphoton interaction Hamiltonian is given by $H_{e-p h t}^{i(s)}=\frac{q_{e} \vec{p} \cdot \vec{A}_{i(s)}}{m_{e}} ; \vec{p}$ being the electron quantum momentum, $q_{e}$ the elementary charge and $\vec{A}_{i(s)}$ the potential vector of the incident (respectively, scattered) light. We assume deformation-potential interaction between the electronic states and the LA vibrations $H_{e-v i b}=D_{e(h)}(z) \vec{\nabla} \cdot \vec{u}_{m}(z)$, where $D_{e(h)}(z)$ is the conduction (respectively, valence) band deformation potential. ${ }^{23-27}$

The sum in Eq. (3) runs over all intermediate conduction states $e$ and $e^{\prime}$ and initial valence states $h$. For the sake of simplicity we consider here only zero in-plane wavevector electron-hole transitions. Each state is described by a Bloch wave function $\Psi_{e(h)}(\vec{r})=v_{e(h)}(\vec{r}) \phi_{e(h)}(z)$ where $v_{e(h)}(\vec{r})$ is the atomiclike wave function of the electrons (respectively, holes) and $\phi_{e(h)}(z)$ the envelope wave function along the superlattice axis. The latter is calculated by solving Schrödinger equation numerically and assuming parabolic dispersion of the valence and conduction bands. The validity of this assumption is discussed further. Calculations are performed for the real superlattices: the fact that the SL size is limited by the surface and substrate presence has been taken into account. However, the superlattices length was limited to 40 periods to keep within reasonable computation times. As a matter of fact, we have checked that the results do not to differ significantly when changing the number of periods from 20 to 40 .

In the case where only one electron-hole transition is resonantly excited by the probe light, the sum in Eq. (3) reduces to one dominant term and the RB peak frequencies and intensities are directly related to the spatial distribution of the electronic density selected by the optical excitation. ${ }^{16}$ When considering a large collection of intermediate states, summation of the scattering amplitudes in Eq. (3) leads to strong interferences in the overall RB intensity. Interpretation of experimental data becomes difficult due to the fact that the relation between the $\mathrm{RB}$ spectral features and the excited electronic states is hidden by the interfering scattering paths. ${ }^{9}$

In previous works, ${ }^{15,16}$ by rewriting the inelastic lightscattering efficiency (Eq. (3)), we introduced a RamanBrillouin electronic density which combines the wave functions of the intermediate electronic states according to their incoming and outgoing resonance factors. For a scattering process mediated by the conduction electrons, the normalized RBED is
TABLE II. Damping parameters of the electron-hole transitions $\gamma_{i j}$ and electron/hole deformation potentials $D_{e(h)}$ in GaAs and AlAs. The $i j$ subindices refer to the electron and hole subbands.

\begin{tabular}{|c|c|c|c|c|c|c|}
\hline \multirow[b]{2}{*}{$\begin{array}{c}\gamma_{11} \\
(\mathrm{meV})\end{array}$} & \multirow[b]{2}{*}{$\begin{array}{c}\gamma_{22} \\
(\mathrm{meV})\end{array}$} & \multirow[b]{2}{*}{$\begin{array}{c}\gamma_{13} \\
(\mathrm{meV})\end{array}$} & \multicolumn{2}{|c|}{$\mathrm{GaAs}$} & \multicolumn{2}{|c|}{ AlAs } \\
\hline & & & $\begin{array}{l}D_{e}{ }^{\mathrm{a}} \\
(\mathrm{eV})\end{array}$ & $\begin{array}{l}D_{h}^{\mathrm{b}} \\
(\mathrm{eV})\end{array}$ & $\begin{array}{l}D_{e}{ }^{\mathrm{a}} \\
(\mathrm{eV})\end{array}$ & $\begin{array}{l}D_{h}{ }^{\mathrm{b}} \\
(\mathrm{eV})\end{array}$ \\
\hline 10 & 200 & 500 & 7.17 & 2.72 & 5.64 & 2.61 \\
\hline
\end{tabular}

${ }^{\text {aReference } 26 .}$

${ }^{\mathrm{b}}$ Reference 27 .

$$
\rho_{\mathrm{RB}}^{e}(z)=\frac{1}{\mathcal{R}} \sum_{e, e^{\prime}, h} R_{h, e^{\prime}}^{s} \phi_{e^{\prime}}^{*}(z) R_{e, h}^{i} \phi_{e}(z),
$$

where $R_{e, h}^{i}$ and $R_{h, e^{\prime}}^{s}$ are dimensionless resonance factors given by

$$
\begin{aligned}
R_{e, h}^{i} & =\frac{\left\langle e\left|H_{e-p h t}^{i}\right| h\right\rangle}{E_{i}-E_{e-h}+i \gamma_{e-h}}, \\
R_{h, e^{\prime}}^{s} & =\frac{\left\langle h\left|H_{e-p h t}^{s}\right| e^{\prime}\right\rangle}{E_{s}-E_{e^{\prime}-h}+i \gamma_{e^{\prime}-h}},
\end{aligned}
$$

and

$$
\mathcal{R}=\sum_{e, h} R_{h, e}^{s} R_{e, h}^{i}
$$

is a normalization factor satisfying $\int \rho_{\mathrm{RB}}^{e}(z) d z=1 . \rho_{\mathrm{RB}}^{e}(z)$ is the sum, running over $h$, of the overlapping between the effective wave functions $\Sigma_{e} R_{e, h}^{i} \phi_{e}(z)$, excited at the probelaser energy $E_{i}$, and $\sum_{e^{\prime}} R_{h, e^{\prime}}^{s} \phi_{e^{\prime}}(z)$ giving rise to emission of the scattered photon with energy $E_{s}=E_{i} \pm \hbar \omega_{m}$. The scattering paths involving hole-vibration interactions also contribute to the RB scattering: the corresponding normalized Raman-Brillouin electronic density is given by

$$
\rho_{\mathrm{RB}}^{h}(z)=\frac{1}{\mathcal{R}} \sum_{e, h, h^{\prime}} R_{e, h^{\prime}}^{s} \phi_{h^{\prime}}^{*}(z) R_{h, e}^{i} \phi_{h}(z) .
$$

The overall RB scattering efficiency by a given vibration mode, Eq. (3) plus the contribution from the hole-vibration interaction, may then be rewritten

$$
\left|\mathcal{R} \int\left[D_{e}(z) \rho_{\mathrm{RB}}^{e}(z)+D_{h}(z) \rho_{\mathrm{RB}}^{h}(z)\right] \frac{\partial u_{m}(z)}{\partial z} d z\right|^{2} .
$$

From Eq. (8), $\mathcal{R} \rho_{\mathrm{RB}}^{e(h)}(z)$ appears as the electronic density distribution interacting with the vibration modes and giving rise to the Raman-Brillouin scattering. $\mathcal{R} \rho_{\mathrm{RB}}^{e(h)}(z)$ are complex functions (Eq. (4)) because of the homogeneous broadening of the electron-hole transitions (Table II) and because the photons potential vectors are complex.

One can notice the similarity between Eqs. (1) and (8): $\mathcal{R} D_{e(h)}(z) \rho_{\mathrm{RB}}^{e(h)}(z)$ in Eq. (8) plays the same role as $A_{s}^{*}(z) A_{i}(z) P(z)$ in Eq. (1). Since $\mathcal{R} D_{e(h)}$ is constant and $A_{i(s)}$ is nearly constant within each layer $\left(d_{\text {GaAs(AlAs })} \ll \lambda_{i(s)}\right)$, $\rho_{\mathrm{RB}}^{e(h)}(z)$ can be compared to $P(z)$. However, $\rho_{\mathrm{RB}}^{e(h)}(z)$ includes 


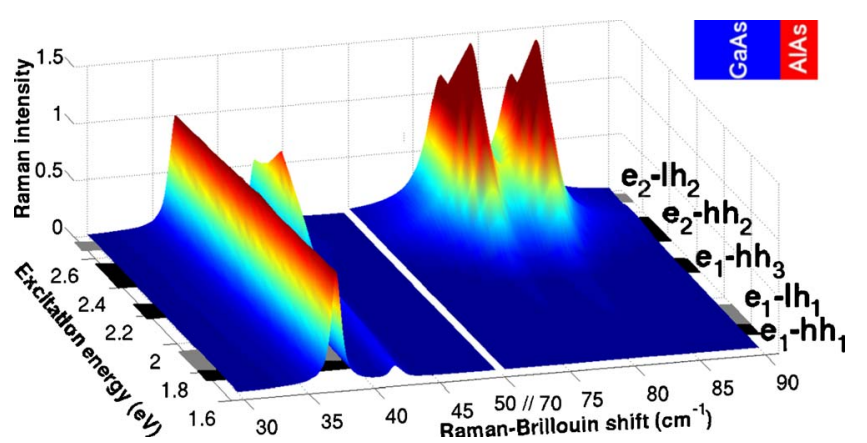

FIG. 3. (Color online) Raman-Brillouin spectra of SL1 calculated using the RBQM and for different excitation energies $E_{i}$. For each excitation energy the scattered intensity is normalized to the first peak $(-1)$. Calculated electron-hole transition energies involving the different subbands are shown on the right.

the electromagnetic fields and the electronic structure of the system and is therefore well suited for the analysis of the resonant light-scattering process. As discussed in Ref. 16, the $\mathrm{RBED}$ is the link between the Raman-Brillouin quantum model and the photoelastic model.

\section{RESULTS AND DISCUSSIONS}

\section{A. Comparison between measured and simulated spectra}

In this section we compare the measured RB spectra to those calculated using the photoelastic model and the Raman-Brillouin quantum model. Our aim is to point out some limitations of the PEM and to show that the RamanBrillouin quantum model gives a more accurate and complete description of the experimental spectra and of their change with excitation energy (resonance effects). This is particularly important because it ensures that the RBED, which will be plotted and discussed in the next section, are indeed those giving a good agreement with experiments.

Figures 3-5 show the Raman-Brillouin spectra of the three superlattices calculated using the RBQM (Eq. (8)) and

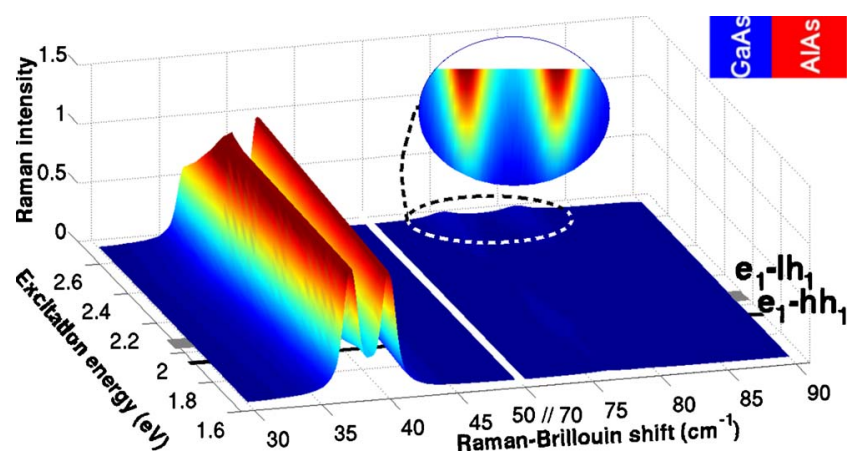

FIG. 4. (Color online) Raman-Brillouin spectra of SL2 calculated using the RBQM and for different excitation energies $E_{i}$. For each excitation energy the scattered intensity is normalized to the first peak $(+1)$. The inset is a focus (color scale multiplied by 20) on the excitation energies for which the small activation of the second doublet $( \pm 2)$ is predicted. Calculated electron-hole transition energies involving the different subbands are shown on the right.

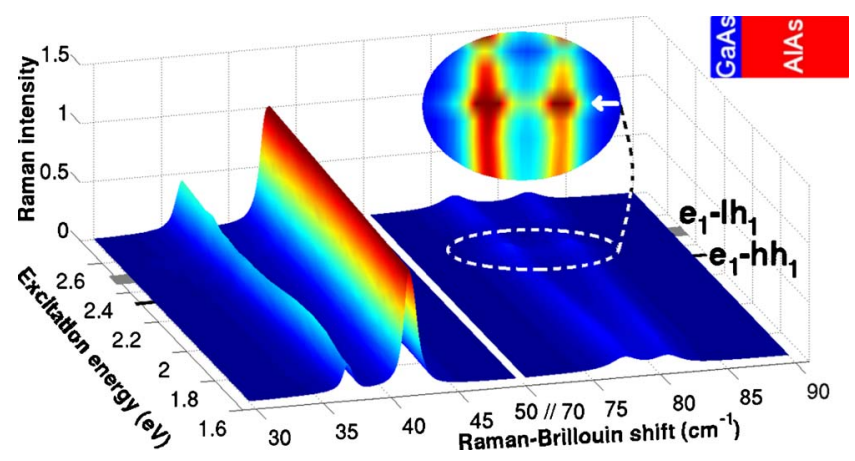

FIG. 5. (Color online) Raman-Brillouin spectra of SL3 calculated using the RBQM and for different excitation energies $E_{i}$. For each excitation energy the intensity is normalized to the first peak $(+1)$. The inset is a focus (color scale multiplied by 20) on the excitation energies for which the broadening of the second doublet $( \pm 2)$ is predicted. Calculated electron-hole transition energies involving the different subbands are shown on the right.

for excitation energy ranging from 1.65 to $2.75 \mathrm{eV}$. The calculated electron-heavy hole and electron-light hole transition energies are indicated in each figure. The optical indices used in these calculations were extracted from ellipsometry measurements and depend on the excitation energy. The electronhole transition dampings used in the simulations as well as the deformation potentials are quoted in Table II. The values for the damping are the ones giving the best agreement with the experimental results and are in agreement with the work of Kushibe et al. ${ }^{22}$

Since the Raman-Brillouin peak frequencies of the $( \pm 1)$ and $( \pm 2)$ doublets are well reproduced by both the PEM and RBQM, the comments will be focused on the scattered intensities and on the comparison with the experiments. For SL1 (Fig. 3), the most remarkable point concerns the scattering by the second doublet $( \pm 2)$. Indeed, the spectrum calculated using the RBQM (Eq. (8)) shows no activation of the second doublet $( \pm 2)$ for red excitation $\left(E_{i}=1.65 \mathrm{eV}\right)$ and up to $2.2 \mathrm{eV}$. This is in a very good agreement with the spectrum measured with excitation at $1.65 \mathrm{eV}$ (Fig. 1): scattering by the second doublet is indeed completely absent. On the contrary, the spectrum calculated using the PEM (lower spectrum in Fig. 1) exhibits scattering by both first and second doublets with similar intensities whatever the excitation energy is (not shown here). Indeed, only small variations in the scattered intensities are expected due to small changes in the exchanged wavevector (see Eq. (1)). Moreover, scattering by the second doublet is clearly activated for excitation at 2.41 $\mathrm{eV}$ and then decreases with increasing energy (Fig. 1). This behavior is well reproduced by the calculated spectra: in Fig. 3 the intensity of the second doublet is maximum at $2.55 \mathrm{eV}$ and then decreases with further increase in the excitation energy. The appearance of the second doublet coincides with the resonant excitation of the electron-heavy hole $e_{2}-h h_{2}$ and electron-light hole $e_{2}-l h_{2}$ transitions (indicated in Fig. 3). For GaAs quantum wells of few monolayers, as those of our SLs, the parabolic band approximation overestimates the electronic subband energies. Therefore the calculated electronic transitions may be larger than the actual ones. This results in underestimation of the interference effects and thus leading 
to an overestimation of the Raman intensity of the second doublet, and could explain why the calculated intensity maximum of the second doublet occurs at higher excitation energy $(2.55 \mathrm{eV})$ than observed $(2.41 \mathrm{eV})$.

Concerning the scattering by the first doublet $( \pm 1)$ the experimental intensity ratio $I_{+1} / I_{-1}$ increases from 0.25 at $1.65 \mathrm{eV}$ to 0.7 at $2.54 \mathrm{eV}$ and then decreases to 0.45 at $2.71 \mathrm{eV}$ (Fig. 1). This variation is also connected with the resonant excitation of $e_{2}-h h_{2}$ transitions and is well reproduced by the simulations based on the RBQM (see Fig. 3).

For SL2, the scattering by the second doublet $( \pm 2)$ is predicted to be very small (with respect to scattering by the first doublet $( \pm 1)$ ) by both the RBQM (Fig. 4) and the PEM (Fig. 1) in agreement with the measurements (Fig. 1). However, the RBQM shows that a small activation is expected for excitation energies close to the $e_{2}-h h_{2}$ transitions $\left(2.88 \mathrm{eV}<E_{e_{2}-h h_{2}}<2.97 \mathrm{eV}\right)$. This is indeed observed experimentally for $E_{i} \approx 2.47 \mathrm{eV}$ (Fig. 1). As mentioned above, the parabolic bands approximation may explain the shift between the calculated and observed excitation energies for this activation.

The scattering by the first doublet shows an inversion of the $I_{-1} / I_{+1}$ intensity ratio that occurs (Fig. 1) when changing the excitation energy from 2.41 to $2.71 \mathrm{eV}: I_{-1} / I_{+1}=0.72$ at $2.41 \mathrm{eV}$ and $I_{-1} / I_{+1}=1.37$ at $2.71 \mathrm{eV}$. This behavior is reproduced by the RBQM even though the intensity ratio is reversed compared to the experimental one. This discrepancy can be explained by the fact that, for SL2, the GaAs and AlAs thicknesses are very similar and very small. Indeed, a difference of only one monolayer between the nominal and the actual average thicknesses can be responsible for the inversion of the $I_{-1} / I_{+1}$ intensity ratio. ${ }^{1}$

For SL3, the RBQM simulations are also in good agreement with the experimental data. First, the measured $I_{-1} / I_{+1}$ intensity ratios are very close to the calculated ones (see Figs. 1 and 5). Even the oscillatorylike variation in the $I_{-1} / I_{+1}$ ratio with excitation energy is well reproduced by the simulations: the measured $I_{-1} / I_{+1}$ oscillates between 0.2 (excitation at $2.45 \mathrm{eV}$ ) and 0.6 (excitation at $2.71 \mathrm{eV}$ ); this behavior is well reproduced by the RBQM.

Scattering by the second doublet $( \pm 2)$ is predicted by the RBQM (Fig. 5). The calculated intensity ratio $I_{ \pm 2} / I_{+1}$ is around 0.2 compared to 0.6 experimentally. It reaches a maximum for excitation around $E_{i}=2.45 \mathrm{eV}$, close to the resonances with the $e_{1}-h h_{1}$ and $e_{1}-l h_{1}$ transitions (see inset in Fig. 5), in agreement with the experiments (Fig. 1). The discrepancy between measured and calculated $I_{ \pm 2} / I_{+1}$ intensity ratio, like for SL1, is mostly due to the overestimation of the electronic subbands energies which weaken the interference effects. Moreover, close to the maximum of the second doublet, a broadening of the $( \pm 2)$ Raman bands is predicted (inset in Fig. 5). This effect can explain the broad band observed experimentally (see Fig. 1) for an excitation at $E_{i}=2.47 \mathrm{eV}$. Since the quantum wells (QWs) of SL3 are very narrow, the symmetries of the deformation fields associated to the acoustic modes are not well defined as in SL1 and SL2. Indeed, a slight shift of the nodes with respect to the center of the QW changes significantly the overlap between the deformation field and the RBED. This leads to a bad selection of the wavevector $q$, resulting in a broadening of the $( \pm 2)$ Raman bands. Such effect appears for the second doublet because the associated deformation fields are strongly varying and are therefore more sensitive as compared to the first doublet.

\section{B. Raman-Brillouin electronic density}

In order to understand better the resonant RamanBrillouin scattering, it is necessary to consider the symmetry of the acoustic vibration modes and their coupling to the optically excited electronic states.

We found, from our band-structure calculations that, according to the GaAs thicknesses, and to the number of periods in the SLs (used for the simulations), about 4500 electron-hole transitions may significantly contribute to the light-scattering process depending on the considered SL (symmetry forbidden transitions such as $e_{1}-h h_{2}, e_{1}-l h_{2}$, $e_{2}-h h_{1}$, and $e_{2}-l h_{1}$ were excluded). Since we consider finite superlattices, there is no translational invariance because of the presence of the surface and of the substrate. Therefore, there is no wave vector conservation in the three interaction steps (Eq. (3)) of the light-scattering process, and thus no (spatial) selection of electronic transitions; wave vector conservation progressively and naturally comes out in our simulations when increasing the SLs length with respect to the optical wavelength (i.e., when translational invariance is recovered). Hence, due to the huge number of possible interfering scattering paths, it is very difficult to connect the electronic structure of the SLs to the changes in the RamanBrillouin spectral features. The Raman-Brillouin electronic density introduced in Sec. III B, allows to overcome this difficulty as it combines the thousands scattering paths into a single effective function.

The RBED generated for electrons (Eq. (4)) and for holes (Eq. (7)) are very similar due to the fact that both are mostly confined within the GaAs QWs (type I SLs). We found only small differences in their spatial extension around the QWs due to their different effective masses. Therefore, we will focus on the RBED generated for the electrons only. Moreover since the RB scattering amplitude is proportional to the $\operatorname{RBED} \mathcal{R} \rho_{\mathrm{RB}}^{e}(z)$ (see Eq. (8)), we will discuss plots of this quantity rather than the normalized $\operatorname{RBED} \rho_{\mathrm{RB}}^{e}(z)$ (Eq. (4)).

Figures 6-8 show the Raman-Brillouin electronic density $\mathcal{R} \rho_{\mathrm{RB}}^{e}(z)$ profiles along the $z$ axis of the three superlattices and for excitation energies ranging from 1.6 to $2.85 \mathrm{eV}$. The real $\mathfrak{R}\left[\mathcal{R} \rho_{\mathrm{RB}}^{e}(z)\right]$ and the imaginary $\mathfrak{I}\left[\mathcal{R} \rho_{\mathrm{RB}}^{e}(z)\right]$ parts of the RBED as well as its modulus $\left|\mathcal{R} \rho_{\mathrm{RB}}^{e}(z)\right|$ are shown. The modulation along the superlattice axis is due to the spatial variation in the electromagnetic fields and to the spatial distribution of the electronic states. The modulus of the RBED is mainly localized in the QWs and reflects the SLs periodicity. The RBED profiles (Figs. 6-8) strongly depend on the excitation energy due to optical resonances with the electronhole transitions involving the different SLs subbands. Moreover, one can notice that for some excitation energies the RBED profiles (real and imaginary parts and modulus) exhibit minima: around 1.7 and $1.9 \mathrm{eV}$ in Fig. 6 for instance. These minima arise from destructive interferences between scattering paths from the $e_{1}-h h_{1}, e_{1}-l h_{1}$, and $e_{1}-h h_{3}$ transi- 

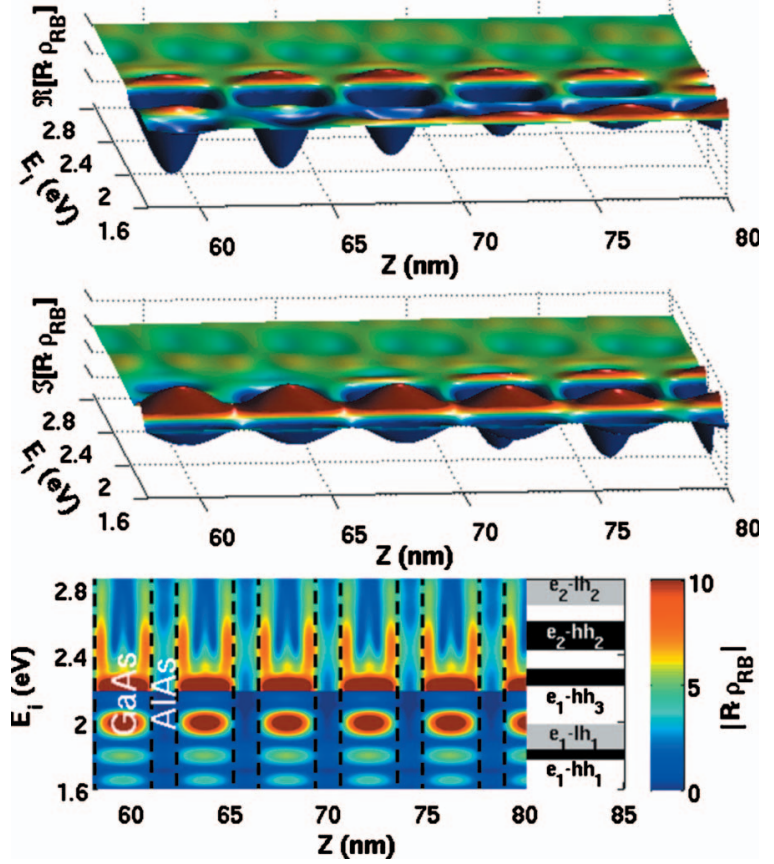

FIG. 6. (Color) RBED $\mathcal{R} \rho_{\mathrm{RB}}^{e}(z)$ spatial distribution for SL1 and for different excitation energies $E_{i}$. From top to bottom are shown the real, imaginary parts and the amplitude of the RBED shown within a few periods of the SL. The color scale of the real and imaginary parts is the same. In the lower panel [modulus of $\left.\mathcal{R} \rho_{\mathrm{RB}}^{e}(z)\right]$, the color scale for energies above $2.2 \mathrm{eV}$ should be divided by 50 . The dashed lines show the barrier/well interfaces. The electron-hole transition energies are reported on the right of the lower panel.

tions. Let us examine for each superlattice how the RBED captures the essential physics of the resonant RamanBrillouin scattering.

For SL1, the RBED profiles (real and imaginary parts and modulus in Fig. 6) exhibit maxima or minima (for the real and imaginary parts) centered on each quantum well for excitation energy $1.8 \mathrm{eV}<E_{i}<2.2 \mathrm{eV}$, i.e., close to resonance with the $e_{1}-h h_{1}, e_{1}-l h_{1}$, and $e_{1}-h h_{3}$ transitions. Therefore, the coupling of the RBED to the acoustic mode $(-1)$ is optimum since the associated deformation field is mainly symmetric with respect to the center of the QWs (see Fig. 2 and Eq. (8)). On the other hand, one can notice from Fig. 2, that the deformation field of the $(+1)$ mode is mainly antisymmetric leading to a weaker overlapping with the RBED. The corresponding Raman-Brillouin scattering is less intense than that of the (-1) mode (see simulated spectra in Fig. 3), in agreement with the measured spectra (Fig. 1).

For excitation close to resonance with the $e_{2}-h h_{2}$ transitions $\left(2.4 \mathrm{eV}<E_{i}<2.6 \mathrm{eV}\right.$ ), the RBED profiles (real and imaginary parts and modulus in Fig. 6) are double peaked with maxima close to the edges of the GaAs QWs. This gives rise to a good overlapping with the deformations $\frac{\partial u_{ \pm 2}(z)}{\partial z}$ associated with the second acoustic doublet $( \pm 2)$ since these deformations are symmetric with two nodes in the QW (see Fig. 2). As a consequence, Raman-Brillouin scattering by the second acoustic doublet is activated (Figs. 1 and 3).

Moreover, scattering by the $( \pm 2)$ doublet does not come out (see Figs. 1 and 3$)$ for red excitation $\left(E_{i}=1.65 \mathrm{eV}\right)$ be-
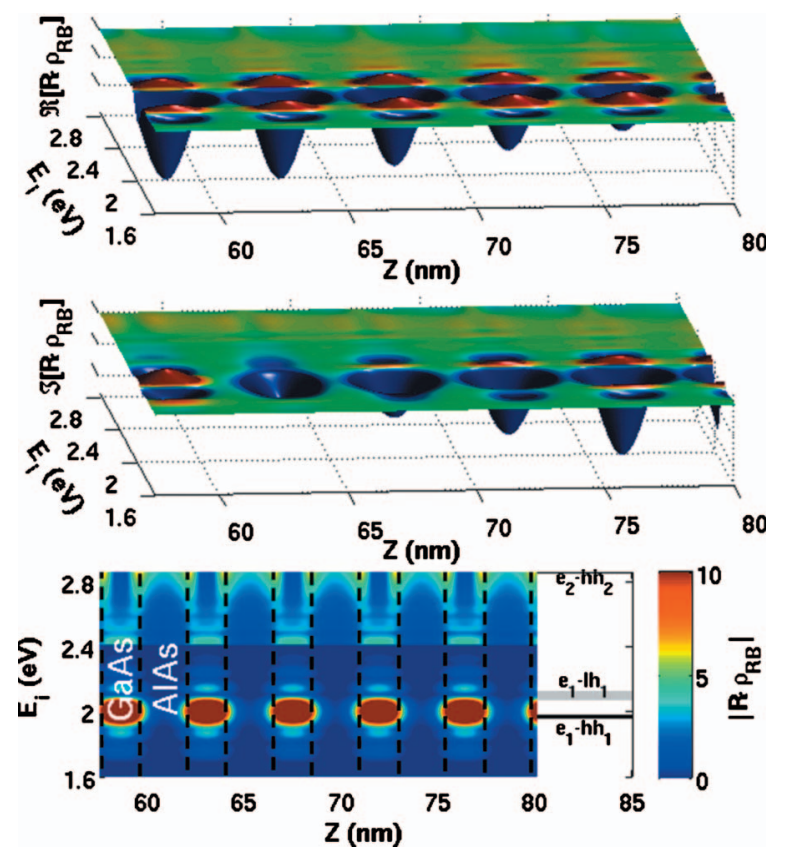

FIG. 7. (Color) RBED $\mathcal{R} \rho_{\mathrm{RB}}^{e}(z)$ spatial distribution for SL2 and for different excitation energies $E_{i}$. From top to bottom are shown the real, imaginary parts and the amplitude of the RBED shown within a few periods of the SL. The color scale of the real and imaginary parts is the same. In the lower panel [modulus of $\left.\mathcal{R} \rho_{\mathrm{RB}}^{e}(z)\right]$, the color scale for energies above $2.4 \mathrm{eV}$ should be divided by 50 . The dashed lines show the barrier/well interfaces. The electron-hole transition energies are reported on the right of the lower panel.
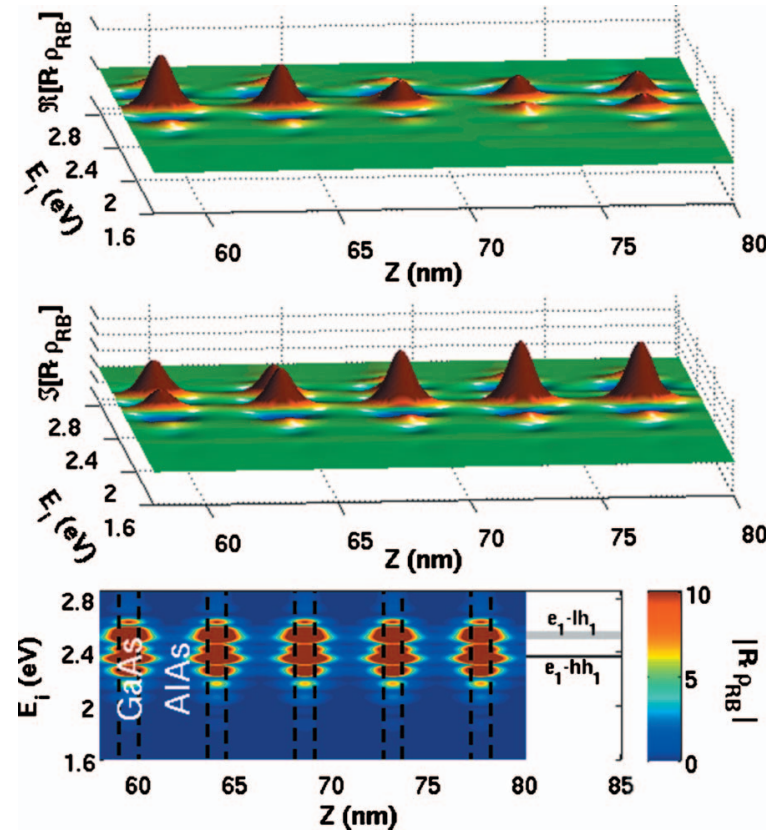

FIG. 8. (Color) RBED $\mathcal{R} \rho_{\mathrm{RB}}^{e}(z)$ spatial distribution for SL3 and for different excitation energies $E_{i}$. From top to bottom are shown the real, imaginary parts and the amplitude of the RBED shown within a few periods of the SL. The color scale of the real and imaginary parts is the same. The dashed lines show the barrier/well interfaces. The electron-hole transition energies are reported on the right of the lower panel. 
cause, although mainly symmetric, the associated deformations has negative and positive values (Fig. 2) that cancel the overlapping with the single peaked symmetric RBED (see Fig. 6).

It is worthwhile to mention that the RBED profiles reported in Fig. 6 point out the reason why the PEM, with a steplike variation in the Photoelastic coefficient (Eq. (1)), fails to describe the Raman-Brillouin scattering in SL1 even for nonresonant excitation (Fig. 1). Indeed, scattering by the second acoustic doublet $( \pm 2)$ is overestimated by the PEM with respect to the experimental data. Due to its steplike shape, the PE coefficient strongly overlaps with the symmetric deformations of the $( \pm 2)$ doublet leading to overestimation of the corresponding Raman-Brillouin intensity.

For SL2, the RBED profiles are symmetric with respect to the center of each GaAs QW. As for SL1, the RBED modulus is maximum at the center of the GaAs QWs for excitation close to resonance with the $e_{1}-h h_{1}$ and $e_{1}-l h_{1}$ transitions whereas for excitation close to resonance with $e_{2}-h h_{2}$ transitions $\left(2.88 \mathrm{eV}<E_{e_{2}-h h_{2}}<2.97 \mathrm{eV}\right)$, the maxima occur close to the edges of the QWs (see real and imaginary parts in Fig. 7). The latter occurs at higher energy than in SL1 because of the smaller GaAs thickness. For energies around $2.8 \mathrm{eV}$, one can notice that part of the RBED is delocalized within the AlAs barriers.

In SL2, the deformation fields of the $( \pm 2)$ vibration modes are mainly antisymmetric with respect to the center of the QWs (Fig. 2). Therefore, their overlapping with the symmetric RBED is very small (in comparison with the case of SL1) and the corresponding Raman-Brillouin scattering is very weak (see Figs. 1 and 4). As noticed in the previous section, a small activation of the $( \pm 2)$ doublet is observed experimentally (Fig. 1) for excitation energy around $2.41 \mathrm{eV}$ and is well reproduced by the simulations (Fig. 4).

Moreover, the change in the $I_{-1} / I_{+1}$ intensity ratio with excitation energy, noticed in the previous section, arises from a slight changes in the symmetry of the $( \pm 1)$ vibration modes: indeed from Fig. 2 one can see that the nodes of the deformation fields are shifted with respect to the center of the GaAs QW so leading to different overlapping with the RBED and to the intensity inversion observed in Fig. 1 when changing the excitation energy.

For SL3 since the QW thickness is only $0.99 \mathrm{~nm}$, there are neither $e_{2}$ nor $h h_{2}$ subbands; the RBED is constructed with $e_{1}, h h_{1}$, and $l h_{1}$ subbands, only. Therefore, the modulus of the RBED, as well as its real and imaginary parts, are symmetric and exhibit a single lobe with respect to the center of the GaAs QW whatever the excitation energy is (Fig. 8). From Fig. 2 one can see that the $(-1)$ vibration mode is mainly antisymmetric with respect to the center of the GaAs QW whereas the $(+1)$ mode is mainly symmetric. Hence, the overlapping of the $(+1)$ mode with the RBED (Fig. 8) is larger than that of the $(-1)$, giving a stronger RamanBrillouin intensity as observed experimentally (Fig. 1) and in the simulations (Fig. 4).

The deformation fields associated with the $( \pm 2)$ modes are mainly symmetric with a single maximum located around the center of the GaAs QW (see Fig. 2). Their overlapping with the symmetric RBED give rise to the activation of the Raman-Brillouin scattering observed in Fig. 1. It is worthwhile to notice that, once again, the PEM overestimates the scattering by the second acoustic doublet with respect to the experimental data (spectra of SL1 and SL3 in Fig. 1) and to the simulation performed with the RBQM (Figs. 3 and 5; red excitation $E_{i}=1.65 \mathrm{eV}$ ).

Finally, it is interesting to mention that according to the spatial distribution of the RBED (Figs. 5-7) in these superlattices, the light-scattering process originates mainly from the GaAs QWs. This is the reason why the empirical assumption $P_{\mathrm{AlAs}} \ll P_{\mathrm{GaAs}}$ has been often used in the PEM to describe the RB scattering in such structures.

\section{CONCLUSION}

In summary we have introduced, here for the first time, the Raman-Brillouin electronic density for semiconductor superlattices. We have shown that plots of the spatial distribution of the RBED along the superlattice axis, combined with the symmetry of the acoustic vibrations, allow for a full understanding of the activation (or the absence) of light scattering for resonant and nonresonant excitation of the optical transitions. The electronic properties of the superlattices were described in the frame of the envelope function approximation and using parabolic dispersions of the valence and conduction bands. Despite this simple model, which becomes questionable for very short superlattice periods, the agreement between measured and simulated RamanBrillouin spectra is satisfactory and the observed resonance behaviors are well accounted for. Comparison and analogy between the profiles of the photoelastic coefficient and of the RBED were presented and discussed. In particular, the impact of the steplike variation in the PE coefficient on the electron-vibrations interaction has been pointed out: it has been shown that because the PE coefficient is constant within each quantum well the electron-vibrations interaction can be overestimated or underestimated depending on the considered excitation energy. This leads to important discrepancies between the spectra simulated using the PE model and the measured ones even for nonresonant excitation, i.e., in a situation where the PEM is supposed to work. Finally, we would like to stress that the approach making use of the RBED as a theoretical tool for the interpretation of the Raman-Brillouin scattering is general. It is very useful when several electronic transitions may be resonantly and simultaneously excited by the probe light. It can be thus extended to a large variety of semiconductor and metallic nanostructures. Moreover since the RBED depends only on the electronic properties it can be used for the analysis of the resonant Raman scattering by optical vibrations as well.

\section{ACKNOWLEDGMENTS}

The authors thank the Department of Industry of the Basque Government through ETORTEK for funding support. 
*bernard.jusserand@insp.jussieu.fr

†adnen.mlayah@cemes.fr

${ }^{1}$ B. Jusserand, D. Paquet, F. Mollot, F. Alexandre, and G. Le Roux, Phys. Rev. B 35, 2808 (1987).

${ }^{2}$ B. Jusserand and M. Cardona, Light Scattering in Solids V: Superlattices and Other Microstructures (Springer, Berlin, 1989).

${ }^{3}$ C. Colvard, T. A. Gant, M. V. Klein, R. Merlin, R. Fischer, H. Morkoc, and A. C. Gossard, Phys. Rev. B 31, 2080 (1985).

${ }^{4}$ R. Merlin, K. Bajema, R. Clarke, F.-Y. Juang, and P. K. Bhattacharya, Phys. Rev. Lett. 55, 1768 (1985).

${ }^{5}$ K. Bajema and R. Merlin, Phys. Rev. B 36, 4555 (1987).

${ }^{6}$ V. F. Sapega, V. I. Belitsky, A. J. Shields, T. Ruf, M. Cardona, and K. Ploog, Solid State Commun. 84, 1039 (1992).

${ }^{7}$ T. Ruf, Phonon Raman Scattering in Semiconductors Quantum Wells and Superlattices (Springer, Berlin, 1998).

${ }^{8}$ P. Y. Yu and M. Cardona, Fundamentals of Semiconductors: Physics and Material Properties (Springer, Berlin, 1996).

${ }^{9}$ M. Giehler, T. Ruf, M. Cardona, and K. Ploog, Phys. Rev. B 55, 7124 (1997).

${ }^{10}$ D. J. Lockwood, M. W. C. Dharma-wardana, J.-M. Baribeau, and D. C. Houghton, Phys. Rev. B 35, 2243 (1987).

${ }^{11}$ D. J. Lockwood, A. H. MacDonald, G. C. Aers, M. W. C. Dharma-wardana, R. L. S. Devine, and W. T. Moore, Phys. Rev. B 36, 9286 (1987).

${ }^{12}$ V. F. Sapega, V. I. Belitsky, T. Ruf, H. D. Fuchs, M. Cardona, and K. Ploog, Phys. Rev. B 46, 16005 (1992).

${ }^{13}$ V. I. Belitsky, T. Ruf, J. Spitzer, and M. Cardona, Phys. Rev. B 49, 8263 (1994).
${ }^{14}$ T. Ruf, V. I. Belitsky, J. Spitzer, V. F. Sapega, M. Cardona, and K. Ploog, Phys. Rev. Lett. 71, 3035 (1993).

${ }^{15}$ J. R. Huntzinger, A. Mlayah, V. Paillard, A. Wellner, N. Combe, and C. Bonafos, Phys. Rev. B 74, 115308 (2006).

${ }^{16}$ A. Mlayah, J. R. Huntzinger, and N. Large, Phys. Rev. B 75, 245303 (2007).

${ }^{17}$ A. Fainstein and B. Jusserand, Semicond. Sci. Technol. 18, S377 (2003).

${ }^{18}$ A. Fainstein and B. Jusserand, Light Scattering in Solids IX: Raman Scattering in Resonant Cavities (Springer, Berlin, 2006), Vol. 15.

${ }^{19}$ J. He, B. Djafari-Rouhani, and J. Sapriel, Phys. Rev. B 37, 4086 (1988).

${ }^{20}$ G. F. Torres de Castillo and I. Rubalcava García, Rev. Mex. Fis. E 52, 172 (2006).

${ }^{21}$ S. M. Rytov, Akust. Zh. 2, 71 (1956) [Sov. Phys. Acoust. 2, 68 (1956)].

${ }^{22}$ H. Kushibe, M. Nakayama, and M. Yokota, Phys. Rev. B 47, 9566 (1993).

${ }^{23}$ J. L. Merz, A. S. Barker, and A. C. Gossard, Appl. Phys. Lett. 31, 117 (1977).

${ }^{24}$ C. Colvard, R. Merlin, M. V. Klein, and A. C. Gossard, Phys. Rev. Lett. 45, 298 (1980).

${ }^{25}$ R. Krönig and W. G. Penney, Proc. R. Soc. London, Ser. A 130, 499 (1931).

${ }^{26}$ C. G. Van de Walle, Phys. Rev. B 39, 1871 (1989).

${ }^{27}$ J. D. Wiley, Solid State Commun. 8, 1865 (1970). 